organizational connectedness in successfully implementing infection-prevention efforts and behavioral change. ${ }^{1}$ How best to sustain effective interventions - perhaps by institutionalizing the interventions - is an area of ongoing investigation.

We agree with Lee and McDonald that a catheter utilization rate of $20 \%$ should not necessarily be used as a performance benchmark in nonICU settings. Currently, there are no national targets for urinary-catheter utilization, although the Centers for Disease Control and Prevention is working on establishing a standardized device-utilization ratio. We have advocated that urinary-catheter use be reported as a quality and performance metric, ${ }^{4}$ given that urinary catheters may have both infectious and noninfectious harms. We have not formally evaluated the potential misclassification of catheter-associated UTI as nosocomial UTI, an interesting concept that requires further study. We completely agree that avoiding the indwelling catheter - whether it is in the emergency department ${ }^{5}$ or the operating room - is important.
Sanjay Saint, M.D., M.P.H.

M. Todd Greene, Ph.D., M.P.H.

Veterans Affairs Ann Arbor Healthcare System

Ann Arbor, MI

saint@umich.edu

Mohamad G. Fakih, M.D., M.P.H.

Ascension Health

St. Louis, MO

Since publication of their article, the authors report no further potential conflict of interest.

1. Damschroder LJ, Banaszak-Holl J, Kowalski CP, Forman J, Saint S, Krein SL. The role of the champion in infection prevention: results from a multisite qualitative study. Qual Saf Health Care 2009;18:434-40.

2. Meddings J, Reichert H, Greene MT, et al. Evaluation of the association between Hospital Survey on Patient Safety Culture (HSOPS) measures and catheter-associated infections: results of two national collaboratives. BMJ Qual Saf 2016 May 24 (Epub ahead of print). 3. Saint S, Fowler KE, Sermak K, et al. Introducing the No Preventable Harms campaign: creating the safest health care system in the world, starting with catheter-associated urinary tract infection prevention. Am J Infect Control 2015;43:254-9.

4. Fakih MG, Gould CV, Trautner BW, et al. Beyond infection: device utilization ratio as a performance measure for urinary catheter harm. Infect Control Hosp Epidemiol 2016;37:327-33.

5. Fakih MG, Heavens M, Grotemeyer J, Szpunar SM, Groves C, Hendrich A. Avoiding potential harm by improving appropriateness of urinary catheter use in 18 emergency departments. Ann Emerg Med 2014;63(6):761-8.e1.

DOI: 10.1056/NEJMc1609988

\title{
Body-Mass Index in Adolescence and Cardiovascular Death in Adulthood
}

TO THE EDITOR: The study by Twig and colleagues (June 23 issue) ${ }^{1}$ adds to our understanding of the long-term adverse effects of obesity in adolescents. However, this observational study cannot establish causation; moreover, the authors did not take into account the potential effects of changes in bodymass index (BMI) over time or during adulthood. This latter point is important, because a reduction in BMI in overweight or obese persons has been shown to improve clinical outcomes. ${ }^{2}$ Furthermore, the authors were not able to adjust for confounders, including physical activity, during the teen years or adulthood, which may markedly affect prognosis. ${ }^{3}$ Simple anthropometric variables of central obesity, such as waist circumference and waist-to-hip ratio, which have been shown to be more closely related to the degree of adiposity and cardiovascular outcomes than BMI alone, ${ }^{4}$ were not assessed. However, despite the limitations, the results of this study are important, because the current prevalence of obesity and physical inactivity among adolescents in the United States is considerably greater than that noted in Israel three to five decades ago and could lead to worse cardiovascular outcomes in the future. ${ }^{3}$

Abhishek Sharma, M.D.

State University of New York Downstate Medical Center

New York, NY

abhisheksharma4mamc@gmail.com

Carl Lavie, M.D.

Ochsner Clinical School

New Orleans, LA

No potential conflict of interest relevant to this letter was reported.

1. Twig G, Yaniv G, Levine H, et al. Body-mass index in 2.3 million adolescents and cardiovascular death in adulthood. N Engl J Med 2016;374:2430-40.

2. Becque MD, Katch VL, Rocchini AP, Marks CR, Moorehead C. Coronary risk incidence of obese adolescents: reduction by exercise plus diet intervention. Pediatrics 1988;81:605-12.

3. Lavie CJ, McAuley PA, Church TS, Milani RV, Blair SN. Obesity and cardiovascular diseases: implications regarding fitness, fatness, and severity in the obesity paradox. J Am Coll Cardiol 2014;63:1345-54.

4. Oliveros E, Somers VK, Sochor O, Goel K, Lopez-Jimenez F. The concept of normal weight obesity. Prog Cardiovasc Dis 2014; 56:426-33.

DOI: 10.1056/NEJMc1609415 
TO THE EDITOR: The finding by Twig et al. that a person's BMI at 17 years of age is positively associated with the risk of cardiovascular death strengthens the evidence that risk factors in early life are related to the development of cardiovascular disease in adults. However, persons who are 17 years of age are more similar to young adults than to children. Thus, key questions remain unanswered relating to the earliest ages in childhood at which an increased risk of cardiovascular disease might be present and at which the risk factors for cardiovascular disease, either in association with or independent of obesity, are arbiters of identifiable cardiovascular disease in adults.

The answers to these questions will require prospective data from childhood and adolescence with linkage to later cardiovascular disease. We are conducting an ongoing collaborative study of the International Childhood Cardiovascular Cohort (i3C) Consortium, ${ }^{1}$ which consists of seven cohorts of more than 40,000 persons with measurement of a broad base of cardiovascular risk factors during childhood and early adulthood. The oldest participants have reached 60 years of age and are being followed for the occurrence of cardiovascular disease. Terence Dwyer, M.D., M.P.H.
George Institute for Global Health
Oxford, United Kingdom
terence.dwyer@georgeinstitute.ox.ac.uk
Alan R. Sinaiko, M.D.
University of Minnesota
Minneapolis, MN
Olli T. Raitakari, M.D., Ph.D.
University of Turku
Turku, Finland
for the i3C Consortium Writing Group
No potential conflict of interest relevant to this letter was re-
ported.

1. Dwyer T, Sun C, Magnussen CG, et al. Cohort profile: the International Childhood Cardiovascular Cohort (i3C) Consortium. Int J Epidemiol 2013;42:86-96. DOI: $10.1056 /$ NEJMc1609415

THE AUTHORS REPLY: Sharma and Lavie record limitations that we delineated in our article that relate largely to the lack of measures of BMI in adults and the lack of data regarding potentially important confounders. We agree that a single observational study cannot establish causation - one must judge the total body of evidence. Clearly, serial measurements of BMI across the course of life would be informative in unraveling whether measures obtained during adolescence predict outcomes independently of measures obtained during adulthood and in assessing the effects of weight change in both directions, especially given conflicting results. ${ }^{1,2}$ The possibility of confounding, particularly by baseline smoking and exercise and their changes over time, exists. Nevertheless, our adjustments for educational level, socioeconomic status, and ethnic origin, which were strongly associated with smoking in a subgroup of our cohort, ${ }^{3}$ account in part for smoking. Other investigators (cited in our article) have found that the association of BMI and cardiovascular outcomes persisted when accounting for smoking and - by mendelian randomization - for overall confounding. ${ }^{4}$

Measures of central obesity may better capture data on adiposity and may better predict cardiovascular outcomes, which suggests that the strong associations of BMI with cardiovascular mortality that were evident in our cohort may be underestimated. BMI remains an informative, simple, available, and commonly used measure in clinical and epidemiologic settings and, as such, enables comparison across studies.

We concur with Dwyer et al. that the association between $\mathrm{BMI}$ in adolescents and cardiovascular risk cannot simply be extrapolated to earlier childhood, and we await their findings. Nevertheless, when one assesses the continuum of childhood and adolescence in a Danish cohort of children $^{5}$ and our cohort, there is support not only for the supposition that the high risk associated with adiposity is identifiable in childhood ${ }^{5}$ but also for the finding that the association between BMI and cardiovascular risk appears to become stronger from childhood to early adolescence ${ }^{5}$ and late adolescence, as seen in our cohort.

Future studies in the field may fill important gaps. Our finding of an association above the 50th percentile of BMI requires confirmation in large cohorts, but it suggests that a rethinking of the "normal range" is in order.

\section{Gilad Twig, M.D., Ph.D.}

Israel Defense Forces Medical Corps

Tel Hashomer, Israel

gilad.twig@gmail.com

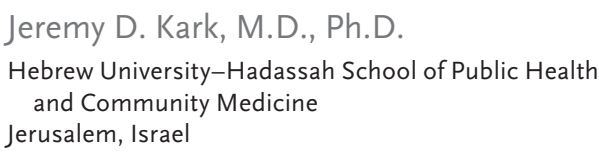


Since publication of their article, the authors report no further potential conflict of interest.

1. Juonala M, Magnussen CG, Berenson GS, et al. Childhood adiposity, adult adiposity, and cardiovascular risk factors. N Engl J Med 2011;365:1876-85.

2. Tirosh A, Shai I, Afek A, et al. Adolescent BMI trajectory and risk of diabetes versus coronary disease. N Engl J Med 2011;364: 1315-25.

3. Kark JD, Laor A. Cigarette smoking and educational level among young Israelis upon release from military service in 1988 — a public health challenge. Isr J Med Sci 1992;28:33-7.

4. Würtz P, Wang Q, Kangas AJ, et al. Metabolic signatures of adiposity in young adults: Mendelian randomization analysis and effects of weight change. PLoS Med 2014;11(12):e1001765. 5. Baker JL, Olsen LW, Sørensen TIA. Childhood body-mass index and the risk of coronary heart disease in adulthood. N Engl J Med 2007;357:2329-37.

DOI: 10.1056/NEJMc1609415

\section{Sudden Cardiac Death in Children and Young Adults}

TO THE EDITOR: The findings of Bagnall et al. (June 23 issue) ${ }^{1}$ about the role of genetic testing in cases of sudden cardiac death deserve comment. The high incidence of cases of sudden cardiac death in children 1 to 5 years of age that were classified as unexplained (37 of 49 cases) is problematic. Moreover, genetic testing did not help to identify a cause in most of these cases. According to Table S11 in the Supplementary Appendix of the article (available with the full text of the article at NEJM.org), a likely pathogenic variant was identified in only 6 of these cases.

We suggest that testing for variants in the calmodulin genes CALM1, CALM2, and CALM3 should be performed in cases of sudden cardiac death in children 1 to 5 years of age who have normal postmortem test results. Variants in a calmodulin gene were first described as a cause of severe forms of primary electrical disorders and unexplained cardiac death in $2012^{2}$; since then, there has been growing evidence that the aggressive phenotypic manifestations of calmodulin gene variants cause sudden cardiac death in infants with structurally normal hearts. ${ }^{3-5}$ The poor diagnostic yield of genetic testing that was observed in the group of children 1 to 5 years of age would probably be improved with the inclusion of testing for calmodulin gene variants.

Juan Jiménez-Jáimez, M.D., Ph.D.

Miriam Jiménez-Fernández, M.D.

Luis Tercedor, M.D.

Complejo Hospitalario Universitario de Granada

Granada, Spain

jimenez.jaimez@gmail.com

No potential conflict of interest relevant to this letter was reported.

1. Bagnall RD, Weintraub RG, Ingles J, et al. A prospective study of sudden cardiac death among children and young adults. N Engl J Med 2016;374:2441-52.

2. Nyegaard M, Overgaard MT, Søndergaard MT, et al. Mutations in calmodulin cause ventricular tachycardia and sudden cardiac death. Am J Hum Genet 2012;91:703-12.
3. Crotti L, Johnson CN, Graf E, et al. Calmodulin mutations associated with recurrent cardiac arrest in infants. Circulation 2013;127:1009-17.

4. Makita N, Yagihara N, Crotti L, et al. Novel calmodulin mutations associated with congenital arrhythmia susceptibility. Circ Cardiovasc Genet 2014;7:466-74.

5. Marsman RF, Barc J, Beekman L, et al. A mutation in CALM1 encoding calmodulin in familial idiopathic ventricular fibrillation in childhood and adolescence. J Am Coll Cardiol 2014;63: 259-66.

DOI: $10.1056 / N E J M c 1609620$

THE AUTHORS REPLY: We thank Jiménez-Jáimez et al. for their suggestion regarding the testing of variants in the calmodulin genes CALM1, CALM2, and CALM3 in cases of unexplained sudden cardiac death in our study, specifically in cases involving children 1 to 5 years of age. When the study commenced in 2010, the potential role of variants in the calmodulin genes had not been reported. ${ }^{1}$ Therefore, the calmodulin genes were not tested in the initial genetic studies, because we focused on 59 genes that were common to all sequencing panels, and calmodulin genes were not present on commercially available cardiac gene panels. In our study, genetic testing was performed in 113 cases of unexplained sudden cardiac death, and subsequent whole-exome sequencing was performed in 62 of these 113 cases (see Fig. 1 of our article). In these 62 cases in persons 1 to 35 years of age, no pathogenic or likely pathogenic variants in coding regions were identified in CALM1, CALM2, or CALM3 genes. One synonymous variant in CALM1 p.Lys78Lys was identified in a case of unexplained sudden cardiac death in a 35 -yearold South Asian man; there are nine reports of this synonymous variant in South Asian persons in the Exome Aggregation Consortium database. No calmodulin variants were identified in 6 of 37 cases of unexplained sudden cardiac death that occurred in children 1 to 5 years of age for whom exome data were available. 